\title{
¿OBLIGACIÓN MORAL O RESPONSABILIDAD POLÍTICA? LAS AUTORIDADES BORBÓNICAS EN TIEMPO DE EPIDEMIAS EN LA CATALUÑA DEL SIGLO XVIII
}

\author{
Alfonso ZARZOSO* \\ Universitat Pompeu Fabra
}

\begin{abstract}
Resumen
En el marco de un siglo XVIII caracterizado por el crecimiento, este trabajo se centra en el estudio de las enfermedades epidémicas que afectaron a la población de la Conca de Barberá durante la segunda década del siglo XVIII. Primeramente, se evalúa la incidencia de las enfermedades en la evolución de la población, junto a los posibles elementos causales, dentro de un contexto socioeconómico que permite analizar el «marco social de la crisis». En segundo lugar, se estudian las medidas de lucha dictadas por las autoridades, así como el espacio político abierto al ejecutarse aquéllas. Finalmente, se hace una valoración general de estos factores para poder comprender el papel del Estado del siglo XVIII y sus limitaciones.
\end{abstract}

\begin{abstract}
Framed by a century featured by its growth, this work deals with the study of the epidemic diseases that affected the population of Conca de Barberà throughout the years 1720 's. Firstly, the author evaluates the demographic impact of these diseases along with its possible causes inside its socioeconomic context, which allows to analyze the «social frame of the crisis». The measures dictated by the authorities and the following political attitudes that emerged are also studied. Finally, these factors are discussed in order to get a general interpretation about the role of the 18 th-Century State and its limits.
\end{abstract}

La Cataluña del siglo XVIII suele presentarse por los historiadores como un período de importantes cambios en la lucha contra la enfermedad causante de alta mortalidad epidémica. En este sentido, se acostumbra a caracterizar como factores determinantes en los cambios demográficos a las transformaciones políticas y económi-

\footnotetext{
Alfonso Zarzoso. Doctorando en Historia y Becario FPI-MEC en el Institut Universitari d'Història
} Jaume Vicens i Vives de la Universidad Pompeu Fabra (Barcelona). 
cas. Así, la desaparición o ausencia de una enfermedad tan temida como fue la peste junto al desarrollo de una incompleta, pero a menudo efectiva, aplicación de controles y medidas administrativas de prevención epidémica contribuyeron a la reducción de las puntas de mortalidad catastrófica y favorecieron un crecimiento sostenido de la población. De esta forma, sería interesante analizar los estudios que han intentado definir y comprender en su contexto esta nueva política administrativa de prevención, surgida a partir de la peste de Marsella, tanto en el plano estatal como en el local. No obstante los esfuerzos de algunos estudiosos, todavía queda mucho por hacer en ambos ámbitos de trabajo. Por esta razón, he pensado que un indicador de los cambios y actitudes mencionados es el estudio de la respuesta de las autoridades borbónicas en materia de prevención y curación de otras enfermedades infecciosas en un espacio político determinado, fijando la atención en el contexto social y económico en que hicieron presencia tales enfermedades, el carácter de éstas, los efectos de la aplicación de las medidas y las funciones propias de los diferentes grupos que tomaron parte en tal situación en el marco del Antiguo Régimen.

El presente trabajo se centra, por tanto, en el estudio de la epidemia de tercianas desarrollada en el arco geográfico que va desde las tierras de Lleida al Campo de Tarragona. En el marco de tal geografía se ha fijado la atención en diez municipios afectados situados en la Conca de Barberà, a medio camino entre Lleida y Tarragona: Montblanc, Espluga de Francolí, Sarral, Solivella, Guàrdia dels Prats, Lilla, Pira, Blancafort, Barberà y Ollers. Esta epidemia tuvo una incidencia notable, aunque intermitente, entre los años 1724 y 1729.

La lectura de la bibliografía local centrada en este período es importante para poder unir los diferentes elementos explicativos y llegar a una comprensión general del fenómeno analizado. En cuanto a las fuentes de archivo utilizadas, si bien cabe recordar que la investigación forma parte de una tesis doctoral en curso de elaboración, han sido consultados los siguientes fondos: Archivo de la Corona de Aragón (ACA), Institut Municipal d'Història de Barcelona (IMHB), Archivo Histórico Nacional (AHN) y Archivo Histórico Provincial de Tarragona (AHPT). Aún están sin consultar los registros de correspondencia y los fondos notariales conservados en el Archivo Histórico Archidiocesano de Tarragona. Respecto a las fuentes locales, se debe mencionar la pérdida de la mayor parte de la documentación de este período, según lo conservado en el Archivo Histórico Comarcal de Montblanc. Para el análisis de las series demográficas, construidas a partir de los libros sacramentales de los pueblos estudiados, se ha utilizado el material publicado por historiadores de la población o de la medicina.

\section{APARICIÓN Y EXTENSIÓN DE LAS ENFERMEDADES}

\subsection{Indicadores demográficos}

Las etapas que caracterizan la demografía catalana del siglo XVIII revelan la duplicación de la población en setenta años. En un primer momento, hasta la década de 1740 , si bien se dieron fuertes variaciones intermedias que mostraron la vigencia de las violentas mortalidades periódicas, como la de 1725-1727, el balance resultó positivo y fijó las bases del crecimiento posterior. Resulta importante situar esta punta de 
mortalidad en su ámbito geográfico para observar si incidió localmente de forma negativa en la tendencia a la recuperación demográfica y económica del período (Vilar, 1962: II, 96-99). Aquí sólo se exponen los datos demográficos, inéditos, hallados en la documentación y referentes a los pueblos de la Conca de Barberà que experimentaron las enfermedades. Por lo tanto, se dejan a un lado, de momento, todas aquellas evidencias cuantitativas encontradas relativas a las enfermedades del resto del arco geográfico de estudio.

La evolución de la población de la Conca de Barberà ha sido objeto de investigación en los últimos años por parte de los historiadores de la población. El estudio de los registros parroquiales se ha traducido en análisis específicos dirigidos a confirmar el crecimiento de la población del siglo XVIII. Estas investigaciones han mostrado la existencia de lagunas difícilmente superables a causa de la desaparición o carencias de los libros sacramentales '. En aras de superar tales faltas, algunos estudiosos han intentado utilizar fuentes alternativas para poder establecer el volumen de la población en el siglo XVIII: utilización de censos, levas militares, catastros (Porta, 1979: 41-47).

Con respecto a los datos que presentamos, hay que recordar que si bien no tienen una finalidad fiscal en el sentido de proceder de un objetivo recaudador, sí la tienen en el sentido de que los procuradores de los datos (autoridades locales, certificaciones médicas, de rectores y de escribanos) compartían el interés general de la población en exagerar la realidad con la idea de conseguir la reducción o liberación del pago del catastro y de otras imposiciones. Así, los datos se deben tomar con prevenciones semejantes a las asumidas ante fuentes de tipo fiscal. A pesar de esto, la verificación y comprobación de algunos datos respecto a los ofrecidos por los registros parroquiales dan validez a la información presentada como tendencia próxima a la realidad. En este sentido, resulta importante señalar que las ideas principales que se pretenden mostrar con estos datos son la persistencia de las enfermedades en el tiempo y el importante volumen de enfermos y convalecientes que comportaron. Aspectos que más adelante se situarán en un contexto de causalidad que llevará a plantear la incapacidad de las autoridades borbónicas en el momento de hacer efectivas las intenciones que en materia de policía sanitaria se irrogaron en el año 1720 .

Los datos que nos ofrecen los informes realizados por la comisión de la Real Audiencia han sido comparados con los recuentos fechados en los años 1708 y 1718 (ver Tabla 1). Como se puede observar, la evolución de la población a lo largo de estos años en las distintas localidades afectadas muestra un ligero retroceso en números absolutos que, en definitiva, apenas se aleja del estado de estancamiento de la zona. Al hablar, posteriormente, de los factores determinantes de las enfermedades se podrá entender el estancamiento de la población de la zona estudiada dentro de un marco general de crisis. La tabla 1 indica que las epidemias que afectaron a los pueblos de la

1. Los libros existentes para el período estudiado de estos municipios son: Montblanc (libro de bautismos desde 1734 y completo el de óbitos), Sarral, Blancafort, Lilla y Guàrdia (ninguno), Ollers (no constan), Barberà, Pira y Solivella (libro de bautismos y de óbitos para todo el período) y Espluga de Francolí (libro de bautismos desde 1694, de óbitos desde 1730 y de matrimonios desde 1693). Vid: Grau et al. (1987), Juliano (1987) y Calbet y Vallribera (1990). 
Conca no se destacaron por una mortalidad catastrófica, sino que se encajaron en un contexto de crisis que dificultaba el crecimiento de la población y que solamente fue superado a partir de la aplicación progresiva de las medidas adoptadas para su restablecimiento. En cuanto a la mortalidad de los años 1725-1727, el análisis por localidades muestra coincidencia con los registros parroquiales de óbitos de Solivella y gran aproximación con los de Pira, mientras que en Montblanc fueron añadidas las 56 muertes de 1724 a la agrupación del trienio 1725-1727 y en Barberà resulta un volumen completamente alejado de los datos parroquiales. La ausencia de registros parroquiales impide contrastar el resto de datos para las demás poblaciones de la Conca de Barberà ${ }^{2}$.

Por otra parte, si bien las enfermedades se desarrollaron desde mediados de 1724, los datos de los que disponemos sólo permiten un acercamiento descriptivo y desagregado para dos momentos de 1727, separados por más de medio año (ver Tabla 2). Se desconocen, por tanto, las proporciones de letalidad y mortalidad de las enfermedades, así como su incidencia para los años inmediatamente anteriores. En cualquier caso, si nos atenemos a los datos disponibles para este período, se pueden caracterizar algunos de los elementos de estas enfermedades. Así, cabe señalar, en primer lugar, los elevados porcentajes de menores de diez años afectados. Si bien tampoco escapó de estos altos porcentajes la población adulta de determinadas poblaciones (Solivella, Guàrdia, Blancafort, Barberà), teniendo en cuenta que ambas columnas corresponden al total de la población de cada lugar, se puede concluir destacando la magnitud de la población infantil susceptible de caer enferma. Esta situación se corresponde con los porcentajes de mortalidad infantil respecto a la adulta y al total de la población, según los registros parroquiales existentes. Las cifras totales comparadas de la prevalencia temporal de las enfermedades a lo largo del año 1727 permite destacar su persistencia. La dilación que caracterizó la puesta en funcionamiento de las medidas de lucha produjo, por tanto, que las epidemias se cebaran entre la población, enfermándola, debilitándola y retrasando su recuperación.

Se debe subrayar también el importante volumen de enfermos pobres de solemnidad y, por consiguiente, sin recursos para superar la crisis. Por ello, no sin razón algunas de las medidas dictadas por las autoridades estaban directamente dirigidas a su recuperación. A finales del año 1727 representaban los enfermos pobres un $21,7 \%$ del total de enfermos de la Conca, mientras que constituían todavía un $17,4 \%$ de los enfermos existentes en el año 1729, aunque por entonces se había reducido el número total de enfermos a 218 personas. Con todo, su dimensión lleva a resaltar los casos de Sarral y Lilla, donde los enfermos pobres significaban más de una tercera parte de la población y su totalidad, respectivamente, en relación con el número total de personas afectadas.

El análisis por localidades (tabla 2) muestra que, si bien las poblaciones más grandes (Montblanc, Espluga de Francolí y Sarral) estaban fuertemente castigadas por las enfermedades, fue precisamente en las menos pobladas donde aquéllas resultaron ser más graves. De tal forma, a excepción de Lilla, entre una y dos terceras par-

2. Para una comprensión mayor del contexto de esta crisis, además de los trabajos citados anteriormente sobre los registros parroquiales, véase Grau (1990) y Gual (1994). 


\section{TABLA 1}

Evolución de la población de las localidades afectadas de la Conca de Barberà durante el primer tercio del siglo XVIII

\begin{tabular}{|c|c|c|c|c|c|}
\hline $\begin{array}{l}\text { CONCA } \\
\text { DE } \\
\text { BARBERÀ }\end{array}$ & $\begin{array}{c}1708 \\
\text { CASAS }^{2}\end{array}$ & $\begin{array}{c}1727 \\
\text { VECINOS }^{n}\end{array}$ & $\begin{array}{c}1718 \\
\text { HAB. }\end{array}$ & $\begin{array}{c}1727 \\
\text { HAB. }\end{array}$ & $\begin{array}{c}\text { 1725-27 } \\
\text { MUERTES }\end{array}$ \\
\hline Montblanc & 275 & 242 & 1.115 & 968 & 256 \\
\hline Espluga & 167 & 156 & 663 & 624 & 133 \\
\hline Sarral & 196 & 234 & 929 & 936 & 60 \\
\hline Solivella & 74 & 56 & 262 & 224 & 45 \\
\hline Guàrdia & 40 & 28 & 130 & 112 & 44 \\
\hline Lilla & 32 & 25 & 88 & 100 & 10 \\
\hline Pira & 30 & 22 & 83 & 88 & 14 \\
\hline Blancafort & 69 & 55 & 312 & 220 & - \\
\hline Barberà & 70 & 67 & 324 & 268 & 7 \\
\hline Ollers & 8 & 8 & 50 & 32 & 2 \\
\hline
\end{tabular}

a. Josep Aparici (1708) Descripción y Planta del Principado de Cataluña, (Iglésies, 1974: I, 78-80).

b. ACA, RA, Consultas, Reg.139, B.12/4/1727 Informe de la RA a SM.

c. Relación General de el Vezindario de el Principado de Cataluña formado en el año 1718 (Vilar, 1962: II, 18-29 y 145).

d. ACA, RA, Consultas, Reg.139, B.12/4/1727 Informe de la RA a SM.

e. ACA, RA, Consultas, Reg. 139, Montblanc.17/10/1727 Comisión RA a Capitán General.

TABLA 2

Prevalencia estacional $(\%)$ de las enfermedades a lo largo de $1727^{\text {a }}$

\begin{tabular}{|lcccc|}
\hline $\begin{array}{l}\text { CONCA } \\
\text { DE } \\
\text { BARBERÀ }\end{array}$ & $\begin{array}{c}\text { Población } \\
-\mathbf{1 0} \text { años }\end{array}$ & $\begin{array}{c}\text { Población } \\
+10 \text { años }\end{array}$ & $\begin{array}{c}\text { TOTAL } \\
\text { ABRL }\end{array}$ & $\begin{array}{c}\text { TOTAL } \\
\text { OCTUBRE }^{c}\end{array}$ \\
\hline Montblanc & 6,8 & 16 & 23 & 11 \\
\hline Espluga & 6 & 14 & 20 & 37 \\
\hline Sarral & 13 & 21 & 34 & 31 \\
\hline Solivella & 13 & 29 & 43 & 4 \\
\hline Guàrdia & 9,8 & 33 & 43 & 58 \\
\hline Lilla & 3 & 13 & 16 & 31 \\
\hline Pira & 54 & 22 & 77 & 22 \\
\hline Blancafort & 19 & 28 & 47 & - \\
\hline Barberà & 16 & 33 & 50 & 47 \\
\hline Ollers & 12 & 25 & 37 & 34 \\
\hline
\end{tabular}

a. Elaboración propia a partir de los datos ofrecidos en las fuentes citadas en (b) y (c).

b. ACA, RA, Consultas, Reg.139, B.12/4/1727 Informe de la RA a SM.

c. ACA, RA, Consultas, Reg.139, Montblanc.17/10/1727 Ministros RA a Capitán General. 
tes de la población total del resto de lugares estuvo afectada por la epidemia. Este hecho repercutiría probablemente en la recuperación demográfica y económica posterior de estos pueblos. En efecto, las gráficas de población de Barberà, Pira, Espluga de Francolí, Montblanc y Solivella indican un crecimiento negativo entre los años 1724 y 1729 . De hecho, la persistencia de las enfermedades se dejó notar también en el estancamiento de los matrimonios y de la natalidad, según los registros parroquiales. Las curvas de mortalidad comienzan a descender desde el año 1727 y, a partir de diferentes ritmos, se observa en el siguiente trienio una recuperación demográfica en función del aumento del número de matrimonios y de nacimientos?

\subsection{Factores determinantes de las enfermedades}

La aparición y extensión de las enfermedades epidémicas desde el verano de 1724 en Montblanc y otras poblaciones de la Conca de Barberà se deben de entender no sólo dentro de un contexto geográfico más amplio, sino también a partir de la interrelación entre una larga postguerra, especialmente difícil y con efectos negativos sobre la situación socioeconómica de la población, y una estructura agraria característica de aquella zona. Esto significa que el análisis histórico de las enfermedades epidémicas ha de conceder especial atención al «marco social de la crisis» para poder llegar a explicaciones satisfactorias de ésta. Así, no se puede desvincular el estudio de las enfermedades del contexto social y económico que sacudieron y que se traduce, por tanto, en el conocimiento previo de las condiciones políticas, sociales y económicas del ámbito geográfico de análisis ${ }^{4}$.

A partir del mapa sobre el alcance geográfico de las enfermedades, se pueden observar tanto su intermitencia como su irregular presencia espacial. Sin embargo, en conjunto ofrecen una imagen regular y global, de tránsito, en toda una zona integrada desde un punto de vista socioeconómico. Una forma de comprobar esto consiste en averiguar qué tipo de enfermedades experimentaron las distintas zonas señaladas. A través de la costumbre secular de las autoridades de depositar su confianza en el conocimiento de los profesionales sanitarios, los informes médicos realizados por éstos, como certificación de las enfermedades, permiten apuntar su identificación, así como las causas y orígenes probables, a lo largo del período y áreas afectadas.

Veamos en primer lugar la calidad de las enfermedades que afectaron a la población de las tierras de Lleida. Para comenzar, sabemos que el tipo y causas de las epidemias que afligieron a la población de Vallmoll, el Milà y Albarca «eran quartanas nacidas de el escaso y mal alimento por la miseria de [estos] lugares», según los médicos Isidro Pastor y Pedro Bessa ${ }^{5}$. La ciudad, capítulo y gremios de Lleida, reunidos en Junta de Sanidad, comunicaron la existencia de enfermedades a las autoridades

3. En cuanto a las curvas mencionadas, véase Calbet y Vallribera (1990: 42, 53, 57 y 259-265), Grau et al. (1987) y Gual (1994).

4. La referencia al contexto social de la crisis constituye un planteamiento fundamental al proporcionar una nueva forma explicativa de la historia de las mortalidades. Para ello, véase Livi Bacci (1988) y Pérez Moreda (1988).

5. ACA, RA, Consultas, Reg. 130, Barcelona (en adelante, B.), 6/2/1721, Informe sobre las enfermedades de Valmoll y Albarca de la Real Audiencia. AHN, Consejos, leg.11.947, Tarragona, 21/2/1722, Informe de los doctores Isidro Pastor y Pedro Bessa. 
centrales, reconociendo que, «visto el testimonio de los Médicos, se origina la dicha epidemia, aunque no pestilencial sí maligna y pegadiza de unos a otros, de la suma y extrema miseria en que se hallan los Pueblos, y que es transzendente y común esta causa a los demás lugares de Urgell y Garrigas». El documento utilizado por las autoridades centrales y fundamentado en los informes médicos precedentes aún aporta más indicadores de la calidad de las enfermedades. Así, se habla de «calenturas malignas que se pegan y comunican de unos a otros vezinos, prozedidas del hambre $y$ no haver tenido cosecha más de cinco años, ni tener para comprar carne y yelo, que son las cosas más precisas para la curación de estas enfermedades, y de falta de agua». Además, se destaca el papel causal del agua encharcada, ya que «veviendo de élla, aquellos vezinos empezaron las enfermedades, y los Médicos declaran que no remediándose tan suma miseria, perseveran, pues los que escaparen o an de morir de flaqueza o an de recaer por falta de alimentos, y que siendo esta necesidad común en todo el Urgell es de temer pasen a otros lugares estos males» ". El médico Josep Miranda afirmaba que eran «tercianas y cuartanas... [que] son comunes todos los años en este país». Y en uno de los informes finales se apunta que van templando y que «a proporción que vaya refrescando el tiempo y zesando el motivo de los arrozes se reducirán aquellas enfermedades, que las más se han convertido a tercianas, muy ordinario accidente en aquellas partes, como en todas las que se siembran y coxen arrozes $\gg^{7}$. De esta forma, se clarifica el origen de la infección en los pueblos de la ribera del Segre, donde el agua era utilizada, entre otras cosas, para el cultivo de arroz, cáñamo y ciertas legumbres. También se hace evidente la preocupación de las autoridades de Lleida, quienes en una situación parecida pero fuera de la presente acotación cronológica, señalaban «que las aguas infectas que salen de los Arrozes de aquel término [de Térmens], se hechan (sic) y van a parar, igualmente que las de los otros lugares, en el río Segre, de cuias aguas es preciso bever los vezinos de esta Plaza y Tropa de su guarnición, en todo el año, por carecer de otra de buena calidad» ${ }^{*}$.

A continuación, siguiendo el mapa de las enfermedades, nos fijamos en las del Baix Camp, en Tarragona. Para dar una idea de los «sínthomas y señales», los médicos comisionados por la ciudad de Tarragona infirieron que se trataba de una «constelación con calenturas compuestas o complicadas, llamadas con especialidad semitercianas, o complicadas o hemitretens (sic), que resultan de la fiebre continua con intermitente». Consideraban la enfermedad contagiosa, pero no maligna, «en unos parótidas y en otros exhantemas, cólicas, delirios, diarreas, disenterías, opresión y debilidad de pulsos». No dudaban, como seguidores de las teorías hipocráticas, que el aire era «la causa mediata y principal», aunque también hacían partícipe a «la grande abundancia de llubias e inundación de las riberas [que] han podido viciar las aguas que servían comúnmente para el abasto. [...] Concurriendo asimismo las de-

6. AHN, Consejos, leg. 1 1.947, Lleida, 7/8/1722, Memorial elevado al rey por la ciudad de Lleida, basado en los informes de diversos médicos (Josep Miranda, Jacob Domènech, Pere Macip); y Madrid, 18/8/1722, Carta de Luis de Miranda a Josep Rodrigo.

7. AHN, Consejos, leg. 11.947, Lleida, 1/11/1722, Informe del doctor Josep Miranda; y B. 5/12/1722, Informe del Capitán General al Consejo de Castilla.

8. ACA, RA, Papeles de Su Excelencia, Reg. 263, Lleida, 24/9/1732, Carta del Corregidor de Lleida al Capitán General. 
más cosas no naturales, con especialidad los alimentos y pasiones de ámimo, que después del ayre, una vez pervertidos de su orden natural son lo que tienen más actividad a disponer los cuerpos para el contagio». Por lo tanto, un cuadro general seme-

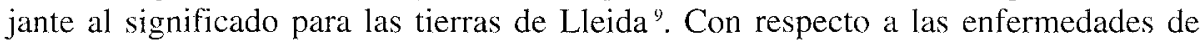
Montblanc -centro comarcal y probable punto de irradiación y propagación de la epidemia al resto de poblaciones de la Conca de Barberà-, ya en el año 1724 se advirtió «que la miseria que padecían con las passiones de ánimo y lo infecto de las aguas, que de diferentes pantanos contiguos a la Villa se comunican a los pozos de que beven, [era] causa de la Epidemial contellación de las tercianas». La reducción y aumento de la presencia de la enfermedad a lo largo del tiempo se atribuía a la «falta de buenos alimentos en dicho tiempo y havría sido causa de que tomassen mayor cuerpo». La perseverancia de la epidemia llevo a la Real Audiencia, para evaluar la situación, a comisionar a un oidor de la institución, acompañado por los doctores Sebastián Creagh, entonces teniente de Protomédico del Principado, y Rafael Esteve, prestigioso médico barcelonés. Ambos profesionales, junto a los médicos de Montblanc, después de reconocer las balsas de agua empozada, informaron que «eran muy perjudiciales a la salud pública de aquel Pueblo, y podría haver sido la causa principal de las enfermedades que en él y en sus lugares circunvezinos se han experimentado y experimentan» ${ }^{10}$.

La caracterización de las enfermedades a partir de los informes médicos, así como su cuadro clínico y su persistencia o estacionalidad, nos lleva a considerarlas -sin ánimo de afirmaciones definitivas - como fruto de la probable combinación de tercianas o cuartanas (paludismo) y fiebres de origen entérico y transmisión hídrica (posiblemente fiebre tifoidea). Más adelante se podrá verificar, al estudiar las medidas dictadas contra las enfermedades, la identificación que se hizo de aquéllas. Otro elemento que cabe destacar desde un punto de vista conceptual es la utilización que hacemos de los términos endemia y epidemia. Así, a causa del frecuente cultivo de arroz y de la intensa acción de empozar cáñamo en las tierras de Lleida y determinadas zonas del Camp de Tarragona, consideramos que estas tercianas eran endémicas y relativamente estables. Y así fue mientras no se dictaron medidas encaminadas a la prohibición o control de tales cultivos y a la mejora de las infraestructuras sanitarias medioambientales. Por otra parte, el carácter de las enfermedades desarrolladas en la Conca de Barberà nos lleva a hablar de enfermedades epidémicas a causa de su origen, persistencia y erradicación.

Los informes médicos proporcionan una serie de claves explicativas que se deben atender: miseria -que se acostumbra a asociar con subalimentación y alimentos en mal estado- contagiosidad, estacionalidad y perseverancia de las enfermedades, cultivo de arroz, balsas de agua y consumo de agua contaminada. Otro elemento des-

9. AHAT, Libros Capitulares, Reg.220, Tarragona, 15/9/1727, Informe realizado por los doctores Antoni Gavaldà, de Reus, y Pau Miquel Carbonell, de Tarragona.

10. ACA, RA, Consultas, Reg.139, B.12/4/1727, Memorial elevado al rey por la Real Audiencia. Paralelamente al inicio de las enfermedades en Montblanc, algunos pueblos del Alt Penedès -Sant Quintí de Mediona y Torelles de Foix-, del Baix Camp -Capafonts-y del Alt Camp-Vila-rodona y el Pont d'Armentera- se vieron afectados por enfermedades parecidas: ACA, RA, Acordadas, reg. 12 , Madrid, 4/11/1724 carta del Consejo de Castilla al Capitán General. 
tacado, desarrollado más adelante, son las malas cosechas, que han hecho hablar de crisis en la inmediata postguerra. Estos aspectos muestran con claridad que el consumo de agua y de alimentos infectados se convirtieron en el mecanismo de transmisión al producirse el contagio fecal-oral, hecho que está en el origen entérico de la fiebre tifoidea. Hay que subrayar también la presencia de un grupo de alto riesgo en la propagación de enfermedades, el ejército. Sabemos que las guarniciones no sólo estaban rodeadas de campos de arroz y por tanto de balsas de agua estancada, sino que los soldados participaban como el resto de la población en el consumo del agua contaminada. A tal presencia militar, que se reveló en una ocupación constante, se debe asociar su movilidad entre Tarragona y Lleida ". Las vías de comunicación señaladas en el mapa muestran que la Conca de Barberà se halla a medio camino entre las tierras de Lleida y el Camp de Tarragona. Además, como medida coactiva, las tropas se instalaban durante el tiempo que hiciera falta en las casas y pueblos que se atrasaban en el pago del catastro. Hecho constante en una coyuntura económica crítica hasta la década de $1730^{\prime 2}$. El tránsito y la instalación discrecional del ejército lo convertía, sin duda, en el grupo de riesgo que mejor podía actuar como portador y transmisor de enfermedades infecciosas originadas tanto por los mosquitos vectores de las tercianas de los arrozales como del mecanismo de contagio fecal-oral de la fiebre tifoidea. Hechos que se enmarcan en una larga y difícil postguerra en la que se combinaron los perniciosos efectos de las malas cosechas con otro elemento impuesto por los vencedores, también odiado por los catalanes: la nueva contribución real, el catastro, que se añadía a los antiguos derechos del General confiscados por la Corona ${ }^{13}$.

Una vez descritos el tipo, origen y mecanismos de transmisión de las enfermedades, resulta importante comprender la referencia hecha a la miseria de la población como causa de las enfermedades. En este sentido, se debe señalar que los estudios de demografía histórica han destacado la importancia del «síndrome de miseria», al actuar de forma sinérgica junto a unas condiciones de pobreza y de mala nutrición, para poder explicar así la incidencia y letalidad de la epidemia (Livi Bacci, 1988: 59; Carmichael, 1985: 51-56). Debemos ver, por tanto, si las enfermedades estudiadas cursaron en una situación de dominio de tal «síndrome de miseria» que mantuviese buena parte de la población en el umbral de la pobreza, predisponiendo a los vecinos a ser susceptibles de las enfermedades.

El significado de miseria, una de las claves aportadas por los médicos, contribuye a caracterizar el tipo de crisis de mortalidad al manifestar, como uno de los factores más importantes de propagación y persistencia de las enfermedades, las condicio-

11. Ocupación, destaca N. Sales, «sovint superior als 30 o 35.000 homes, mai no inferior als 20.000 ». También subraya que en Valls «no hi ha any, fins a la meitat de la dècada de 1730, que no s'hi instal-li, almenys sis o set vegades, alguna partida de tropa, ara vuit dies, ara deu o vint». Véase Sules (1989: 428-429).

12. Tal lue la persistencia de estos actos que algunos testimonios de la época resultan sorprendentes, como el de Miquel Anglada (aunque en Fonteta, cerca de La Bisbal d'Empordà) quien escribía que «antes dels paganents tants grans, pesà una plaga de miquelets que continuament la taula sempra estabe parada hi menjant hi bebent sempre», en Soler (1994: 82).

13. Otro testimonio de la época, Sebastià Casanovas, muestra las amenazas sufridas al hablar del catastro: «...y que si no pagàvam nos enviarian una discreció de deu caballs ab un cabo», en Casanovas (1978: $105)$. 
nes previas de disponibilidad para la extensión de aquéllas en el marco de una estructura agraria muy determinada. Distintos elementos llevan a plantear que la crisis desarrollada en la Conca de Barberà desde 1724 no fue una crisis típica de mortalidad de naturaleza mixta provocada por la combinación de crisis agraria-y/o de crisis de subsistencias- y enfermedad, sino que la aparición, prevalencia y letalidad de las enfermedades estaban relacionadas con esta característica estructura agraria ${ }^{1+}$.

Es bien conocido que los años inmediatamente posteriores a la guerra fueron especialmente difíciles para la economía catalana, de malas cosechas y precios altos, traduciéndose en una crisis agraria que se extendió entre 1715 y 1724 y que produjo graves hambrunas, aunque no generalizadas a todo el Principado y con diferentes ritmos de recuperación (Vilar, 1962: II, 387 y ss.; Sales, 1980, III, 46-67). Se puede observar en el mapa la superficie que se corresponde con las 84 poblaciones de la zona triguera (alrededor de Lleida y de otros mercados comarcales fundamentales) que solicitaron el auxilio real en forma de granos para afrontar la siembra y su manutención ante la calamitosa cosecha del año $1722^{15}$. Si bien estas malas cosechas constituían una manifestación de la crisis agraria, tal vez también lo fue el recurso al que optaron los pueblos de la ribera del Segre ante la crítica situación, esto es, el cultivo de arroz. Un cultivo que existía desde siglos, pero que adquirió especial importancia en el primer tercio del siglo XVIII, tanto por el hecho de ser tierras en manos de pequeños propietarios como por su mayor productividad y rentabilidad como substituto de otros cereales, a pesar de las insalubres condiciones que comportaba (Vilar, 1962: II, 284-291; Mateu, 1987: 77-104). Es importante recordar que el cultivo de arroz era la producción «que facilita a los moradores de aquellos Pueblos su subsistencia y la mayor conveniencia para la paga de los cargos a que están precisados». Además, aunque los arrozales podían ser substituidos por otros productos, el mismo informe médico del doctor Joan Pedret, de Reus, reconocía a propósito de esto que «cada jornal de arroz duplica por lo menos el número de quarteras al de judía [y], que de los expresados frutos [judías, maíz, mijo, etc.] es el que da y vale más»" ${ }^{\text {th. }}$

De esta forma, si bien no hay duda sobre la crisis agraria, esto no implica que ésta hubiese dado lugar a una crisis de subsistencias. De hecho, en las tierras de Lleida se ha mostrado que las crisis demográficas no tuvieron, en general, un fundamento de crisis de subsistencias y que los aspectos demográficos de la crisis agraria de postguerra fueron notables sobre la nupcialidad y la natalidad, pero no sobre la mortalidad (Vicedo, 1991: 22-36). De igual forma, los precios del trigo de Montblanc y de

14. En este sentido. Pérez Moreda (1980) ha mostrado que las crisis demográficas del siglo XVIII en la España interior tienen explicación a partir del fundamento epidémico y no de la crisis de subsistencias.

15. Los registros de la sección de Consultas de la Real Audiencia están llenos de referencias en este sentido. A modo de ejemplo, en un informe de la Audiencia al Capitán General sobre la situación política catalana, se dice que «solantente ha ocurrido de nuevo la esterilidad universal de la cosecha de este Principado, para que hecha presente por VEa a SM pueda servir tal vez de motivo para facilitar algun expediente conducente al consuelo y tranquilidad del Principados. ACA, RA. Consultas, Reg.128, B. 21/7/1719, 13v-19v.

16. ACA, RA, Papeles de Su Exc'., Reg. 263, B. 24/4/1732, Intorme de la RA sobre el cultivo de arroz en la ribera del Segre; y Reus, 13/5/1736, Informe del doctor Juan Pedret sobre el arroz en las zonas regadas de Taragona. Para un análisis sobre las relaciones entre el cultivo del arroz y la sanidad y la repercusión económica de esta cosecha en esta zona, véase Andreu (1994: 549-560). 
otros mercados cercanos de distribución no reflejan durante este período repentinos aumentos que expliquen la existencia de una crisis de subsistencias (Garrabou, 1970; Feliu, 1991: 1, 23-30; Grau, 1990: 50-62). No obstante, esto tampoco implica una circulación fluida de subsistencias y, obviamente, aún menos en una coyuntura crítica. No debía ser desmesurado el recelo que tenía el Conde de Montemar en cuanto al efecto que tendrían las cartas dirigidas por el Rey a los señores jurisdiccionales -sólo seis poblaciones eran reales- de los lugares solicitantes de granos, al decir que «quando teniendo con que assistirles los más [de los señores] los dexarán perezer en sus enfermedades» ${ }^{17}$. En este sentido, tal vez se debería relacionar estos problemas con los intentos por parte de la Corona de formar pósitos de granos en el Principado. Política que, como ha mostrado Llimargues (1989), se vería obstaculizada por los intereses económicos de la burguesía comercial. A pesar de ello, es conocido que en situaciones críticas se alzaron voces contra los acaparadores de granos, como fue el caso en la Tarragona de 1728-29 (Vilar, 1962: II, 387). De hecho, a lo largo de este decenio fueron constantes las órdenes dirigidas a las autoridades locales para hacer reservas de granos. Así lo muestra, por ejemplo, la disculpa presentada por el rector de Montiró al Conde de Darnius al no haber podido comprar cereales, «pues en la Era present no és effectuable dita compra, per quant los assentistas del Rey abarcaren dende la cullita tot lo que pogueren, y lo poch que ha quedat lo volen [los campesinos] per resembrar» ${ }^{18}$.

Estos elementos nos llevan a plantear la hipótesis de una precarización en la dieta de la mayor parte de la población. La posibilidad de una precarización en la dieta no implica, sin embargo, escasez o falta de alimentos, como hemos señalado, y tampoco que unos recursos insuficientes fuesen la causa directa de la enfermedad. De hecho, la documentación muestra como enferman o son vunerables a las fiebres personas aparentemente bien nutridas ${ }^{19}$. En cualquier caso, toda ve $z$ que el proceso de precarización nutricional se prolongase a lo largo del tiempo afectaría fuertemente la convalecencia de los enfermos y agravaría, a su vez, la letalidad del proceso de infección. Un indicador, indirecto pero significativo, del grado de nutrición se puede hallar en las medidas dictadas por las autoridades y aconsejadas por los médicos en la lucha contra la enfermedad. Se observa, así, que la medida terapéutica o «de alivio» para los enfermos pobres consistente en el suministro de pan, carne y vino se convirtió, a lo largo de todo el período y de toda la zona geográfica, en una estrategia consolidada de recuperación de los convalecientes. En este sentido, tal y como ha puesto de manifiesto Pérez Moreda (1988: 714-716), no hay duda de que «todo proceso de convalecencia requiere una mayor aportación proteínica y calórica para reponer las reservas

17. AHN, Consejos, leg. 11.947, B. 5/12/1722, Conde de Montemar a José Rodrigo. La lista de todos los municipios junto con la indicación del señor jurisdiccional de cada uno se halla en: IMHB. Sanidad, IV, 3, ff. $51 \mathrm{v}-52 \mathrm{v}$ y $\mathrm{V}, 5$, ff. $186 \mathrm{v}-188 \mathrm{v}$ y $199 \mathrm{v}-200 \mathrm{v}$.

18. ACA, Ordenes militares, 438, Montiró, 16/10/1726, Joan Bta. Caymó a Conde de Darnius.

19. GRAU (1990: 54-55) muestra un informe del Dr. Joan Cuscullana, de 30 de agosto de 1726. donde dice que 19 de los 23 frailes de la comunidad de Montblanc estaban enfermos y también el Alcalde Mayor, el Baile y cuatro de los seis Regidores. Igualmente hallamos a Roxas, el oidor comisionado de la Audiencia, quien a su regreso a Barcelona el mes de noviembre de 1727 advirtió que se encontraba «indispuesto con unas tercianas que traxe de esta commisión». Véase nota 31. 
consumidas en el curso de la infección». Por esto, aparte del grado de eficacia de las medicinas y tratamientos recomendados por los médicos, éstos aconsejaron la bondad de la dieta como la terapia más precisa e inexcusable para el restablecimiento de la salud. Incluso se calculó el gasto diario del enfermo convaleciente en 4 sueldos, por los que debía recibir: «doze onzas de pan bueno y se les abonará por ellas un sueldo..., nueve onzas de carne de carnero bueno y se les pagará un sueldo y ocho dineros.... media quartilla de vino bonificándose por esta razón seis dineros». El resto hasta los 4 sueldos servían para pagar la cama y su ropa, así como las medicinas confeccionadas por el boticario ${ }^{20}$.

Un elemento importante de la estructura agraria que caracteriza a esta zona, la creciente especialización productiva en el área afectada, forma parte de la comprensión general de la persistencia de la enfermedad. Este aspecto se debe unir a los anteriormente descritos para, una vez interrelacionados, pensar en la coyuntura crítica para la mayoría de la población. En efecto, el Campo de Tarragona era deficitario en la producción de cereales e importador de trigo, por la vía marítima y por la terrestre que enlazaba con la zona del Urgell. El déficit era un efecto de la especialización vitivinícola que estaba orientada, a partir de una creciente demanda, a los circuitos interregionales del mercado. Desde el último tercio del siglo XVII se había producido una evolución inversa entre el cultivo de la viña y el del trigo. La Conca de Barberà también desarrolló una evolución productiva semejante, aunque a un ritmo más lento en conjunto, pero acentuado en las poblaciones vecinas a Montblanc. El estudio realizado por Navarro (1984: 727-746), a partir de 15 catastros tarraconenses, ha mostrado que «en los años de abundantes cosechas de trigo y cebada los cereales recogidos no eran suficientes para atender la demanda de un mes de Corregimiento». La viña se convertía así en el cultivo comercial más extendido de la zona, en el elemento económico de apoyo familiar y de la comunidad que primero reaccionaba a los estímulos del mercado, a causa de la necesidad de una fuerte inversión en fuerza de trabajo (Andreu, 1986: 21-31 y 1994: 363-407). De esta forma, la zona estudiada se caracteriza por haber tenido una producción de cereales muy ajustada que, si bien permitía mantener los niveles de subsistencia de la población, la abocaba, en momentos de crisis como el aquí estudiado, a una situación muy crítica.

Se debe recordar, como ha señalado Porta (1986: 48-54), que la mayor parte de la población de la Conca de Barberà $(74,9 \%)$ estaba formada por jornaleros, pequeños propietarios campesinos y artesanos y que la estructura de la propiedad de la tierra mostraba un predominio del muy pequeño propietario y una intensa parcelación $(67,7 \%$ de propietarios con tierras de menos de 5 jornales) junto a un reducido grupo que detentaba la propiedad de grandes superficies $(2,4 \%$ de propietarios con tierras de más de 60 jornales). Esta mayoría de la población vivía en el umbral de la subsistencia y dependía únicamente de su trabajo para pagar los impuestos -como el catastro-, el diezmo y las pensiones de los censales cargados con la idea de mantener el ciclo agrí-

20. Para una explicación de las drogas utilizadas y la función y finalidad de éstas, véase: AHN, Consejos, leg. 1 1.947, Vallmoll. 4/12/1721, Remedios practicados por los Drs. Pau Miquel Carbonell y Mateo Pujol. En cuanto a los detallados planes de las raciones de pan, carne y vino necesarios por enfermo y las correspondientes estimaciones de los importes, véase: ACA, RA, Consultas, Reg. 139, B. 16/10/1727, ff. 164rv, Instrucción de Roxas a los administradores de los hospitales y Jordi (1981). 
cola, comprar semillas, comercializar los pequeños excedentes y subsistir (Ferrer, 1989: 189-212). Si se tiene en cuenta que la situación económica de estas pequeñas explotaciones se hallaba en una situación crítica y de fuertes obligaciones, a causa de los efectos de la guerra, la implantación del catastro en el año 1716 unida a una coyuntura agraria desfavorable abocó a las familias a recurrir a un endeudamiento crónico para poder garantizar la subsistencia de sus miembros. Esta forma de empeñarse se convertía en el mecanismo clave de extracción de renta y afectaría paralelamente a la situación económica de los municipios. En efecto, como se puede hallar en buena parte de la Cataluña Nueva y han advertido Tello (1995: 13-38 y 100-116) y Vicedo (1991: 138-142 y 295-314), estos elementos constituyen las bases de un endeudamiento progresivo de los municipios al hacer constantes repartos, crear censales y firmar concordias con los acreedores para poder hacer frente a los gastos locales ordinarios y extraordinarios.

Fue, por tanto, en esta coyuntura particularmente difícil donde la aparición de las enfermedades, que cursaban con fiebres y debilitación corporal, limitó la capacidad de reacción y defensa de la mayor parte de la población, empobreciéndola y minándola más en tanto que se reducía la capacidad de vender la fuerza de trabajo para sobrevivir. Según esto, es probable que la evolución de las enfermedades no tuviese una incidencia similar para toda la población. En este sentido, sería interesante comprobar en el estudio de los protocolos notariales si se produjeron cambios en la estructura de la propiedad de la tierra durante el período de las enfermedades, debidos a la venta de pertenencias personales o de propiedades a los hacendados locales o foráneos a que tal vez se vio abocada la parte de la población con dificultades para sobrevivir.

\section{EL ESPACIO POLÍTICO: LAS MEDIDAS CONTRA LAS ENFERMEDADES}

Después del fin de la guerra y en el momento de organizar la nueva administración borbónica catalana, las autoridades delegaron las competencias sanitarias en los municipios, que las habían ostentado desde el siglo XIV, pero les privaron de la capacidad jurisdiccional y punitiva. Esta situación cambió sensiblemente con motivo de la amenaza de la peste de Marsella. El estallido de la peste en Francia multiplicó los asuntos preventivos del Consejo de Castilla y ello se tradujo en la creación de una $\mathrm{Su}$ prema Junta de Sanidad del Reino el día 18 de septiembre de 1720. Con esta institución, ha señalado Nadal (1988: 123), «el Estado se ha irrogado una misión de policía sanitaria sobre todo el país» ${ }^{21}$.

La percepción de la población, especialmente en Cataluña, de la misión sanitaria del Estado fue satisfecha por la afluencia de noticias y de medidas preventivas dictadas desde el verano de 1720 y extendidas por todo el Principado. En el contexto de esta política real -basada en el temor a aquello desconocido pero de consecuencias catastróficas recordadas- se ha de incluir la comunicación de la existencia de enfermedades en el Campo de Tarragona. En efecto, todo ello está en la base de la petición de ayuda al Rey. Las respuestas que las autoridades dieron a las peticiones formuladas nos llevan a hablar de un modelo paternalista de protección caracterizado por las

21. Sobre la organización sanitaria ante la enfermedad epidémica en este período, véase Zarzoso (1994). 
medidas de excepción en tiempo de crisis. Un modelo derivado de una tradición secular que comportaba deberes y obligaciones recíprocos, siendo las medidas de ayuda y de auxilio parte fundamental de la moral protectora de los pobres al fomentar su restablecimiento mediante la asistencia en la enfermedad y en la pobreza y el aplazamiento de las obligaciones de pago de tributos y deudas. Estas peticiones y medidas las consideramos como obligaciones morales -de unos en pedir y de otros en ayudarcaracterísticas de una forma determinada de ver el mundo y típicas del Antiguo Régimen. Es decir, la economía moral de los pobres descrita por Thompson (1994:307) al escribir sobre «una visión tradicional consecuente con las normas y obligaciones sociales, de las funciones económicas propias de los distintos sectores dentro de la comunidad».

En relación con las enfermedades de la Conca de Barberà, Arranz (1985: 214216) ha escrito que no tuvieron una gravedad excepcional y que su relevancia documental estuvo causada por la atención librada por las autoridades. Además, ha subrayado, al ver los tipos de ayudas concedidas, la pasividad por la que se distinguió la actitud de las autoridades. Hecho que se explicaría, según dicho autor, a partir de las características no catastróficas de las enfermedades y por tanto incapaces de desestabilizar las bases de la sociedad. Cabe recordar, en este sentido, que las ayudas concedidas por las autoridades desde 1719 hasta 1729-1730 son típicas de una tradición anterior, que los hermanos Peset (1972: 275-375) definieron y estudiaron en el caso valenciano a lo largo del siglo XVIII. Sin duda y a diferencia del Estado actual, que dispone de los medios y que actúa sin imperativos morales, el Estado en formación de Antiguo Régimen, que no disponía de la capacidad ni de los medios necesarios para hacer frente satisfactoriamente a este tipo de problemas, se hallaba en la obligación moral de conservar el orden a partir de determinados actos de presencia que constituían la parte de responsabilidad política que correspondía a tal Estado.

Las peticiones de ayuda por parte de la población a las autoridades reales ante desastres económicos causados por enfermedades y otras catástrofes, solicitando ser auxiliados con granos y con la liberación de impuestos, parecen haber sido frecuentes a lo largo del siglo XVII en el interior castellano, donde no se conoció una desgracia como la peste (Pérez Moreda, 1980: 302-308). Sin duda, la presencia de las autoridades reales y las medidas dictadas eran más notorias ante la amenaza o la existencia de catástrofes como la peste: colaborando en el pago de gastos, renunciando al cobro de derechos, manteniendo las subsistencias básicas a un precio moderado, asistiendo a los pobres enfermos, etc. De hecho, esto es lo que se halla también en la Cataluña del siglo XVII, con la excepción de Barcelona, es decir, la petición de ayuda a las autoridades reales para afrontar la adversidad. Otra cuestión son las dificultades financieras que implicaban estos auxilios y ello se halla de nuevo en el siglo XVIII con la creación por parte de la Corona de una institución sanitaria sin recursos específicos y, por tanto, perpetuadora del localismo en esta materia (Betrán, 1996: 317-338).

El estudio de las enfermedades de la Conca de Barberà nos permite caracterizar los tipos de ayudas solicitadas y las medidas dictadas, así como el juego político que se abrió a partir de ello. Las enfermedades epidémicas se presentaron en Montblanc y pueblos cercanos a finales de 1724 , siguiendo el curso de las vías de comunicación, el concurso de los mercados, etc. La Real Audiencia, que desde el estallido de la peste 
de Marsella se constituyó como Junta de Sanidad del Principado, mantuvo la línea de las actuaciones iniciadas desde 1719. Es decir, informar a la Corte sobre el estado de los pueblos y de las enfermedades, comisionar facultativos para averiguar las causas de aquéllas y procurar la ayuda de los señores jurisdiccionales. A finales de 1724 , Felipe $\mathrm{V}$ ordenó la asistencia a los enfermos y la exención del pago del catastro y otros tributos hasta su pleno restablecimiento. Estos gastos fueron aplicados de los efectos existentes en la llamada «cuenta de sanidad» de la ciudad de Barcelona, creada a raíz de la peste de Marsella. Las enfermedades declinaron durante el verano y otoño de 1725, pero mostraron nueva agresividad en Montblanc durante 1726, «pareciendo que la falla de buenos alimentos en dicho tiempo havría sido causa de que tomassen mayor cuerpo». A partir de la primavera de este año, las enfermedades parecen contagiar a otros pueblos vecinos de la Conca. La gravedad de las enfermedades «huviera sido peor si la piedad de los prelados y otras personas caritativas no huviera, con sus limosnas, sufragado a su necesidad». Es posible que esto explique la ausencia de evidencias documentales (Arranz, 1985: 204-207). A finales de 1726, las enfermedades se hallan presentes en muchos pueblos de la Conca, donde el precario estado de la población parece sobrepasar a la capacidad local para enfrentarse a la adversidad. Por ello, la Real Audiencia escribía que la desgraciada situación «havia originado a la Villa [de Montblanc] su total ruina, pues siendo los cargos del común passadas de diez mil libras de solos censos, de que se deven muchas summas, no llegan a dicha cantidad sus emolumentos fixos; y que siendo no pocas las casas que se arruynan cada año, ninguna se repara, esperando su último exterminio». De esta forma, a fines de 1726, fueron las autoridades locales sindicadas de diez poblaciones de la Conca las que, una vez superadas por la magnitud de la crisis en su competencia municipal, solicitaron ayuda al Rey. A pesar de que con estos elementos ya se podrían discutir los aspectos que entran en juego en momentos de crisis, la cantidad de los datos relativos a las actuaciones llevadas a cabo entre 1727 y 1730 mostrará mejor las características que se quieren destacar ${ }^{22}$.

A pesar de la prohibición de sindicar los bienes de propios municipales sin el permiso de la Real Audiencia, las autoridades locales de la Conca apoderaron a Juan Bautista Bellver para desplazarse a la Corte, «a fin de que buscase en la Real Piedad de nuestro Rey y Señor algún alivio en las miserias que les ocasionan sus enfermedades». Así, a la iniciativa del rector de Sarral se sumaron el resto de las diez poblaciones y, también, el Alcalde Mayor de Montblanc, a través de cartas al Rey en las que expresaban la difícil situación e imploraban la «Real Commiseración» ${ }^{27}$.

A partir de aquí, el Rey, a través del Consejo de Castilla, se interesó por la cuestión y ordenó a la Real Audiencia efectuar informes precisos sobre el estado de las enfermedades y de la población. La Audiencia organizó en el mes de marzo de 1727 una comisión encabezada por su oidor, Gabriel de Roxas, y los médicos Sebastián Creagh y Rafael Steve, quienes debían informar sobre las enfermedades y sus causas

22. En cuanto al producto de los gastos durante estos años y las consideraciones de la Audiencia sobre la situación general, véase: ACA, RA, Consultas, Reg. 139, B. 12/4 y 10/5/1727, ff. 75rv, 113v-115r, Informes de la RA al Capitán General.

23. ACA, RA, Consultus, Reg. 139, B. 16/7/1727, ff. 149r-150v. Informe de la RA; y Villetes, Reg.70. Tarragona, 6 y 13/6 1727, ff. 47v-48r, 59r-60v, Cartas de Bellver. 
y explicar las medidas que se deberían tomar para la curación de los enfermos. La actuación de esta comisión se desarrolló entre el 23 de marzo y el 3 de abril de 1727. Como resultado, libró un extenso y detallado informe en el que se explicaba el número de enfermos, convalecientes y muertos de cada una de las diez poblaciones, así como las rentas de los municipios, las cargas o créditos que se debían satisfacer anualmente, las pensiones atrasadas y el catastro que se pagaba.

Una vez reconocidas las causas de las enfermedades, la comisión aconsejaba la realización de una serie de actuaciones: en primer lugar, se debían terraplenar las balsas de agua estancada que había junto a las murallas de Montblanc. Dado que estas aguas se filtraban en los pozos de las casas, era necesaria la conducción de agua desde una fuente cercana, «cuyo gasto se podría aprontar de las rentas del común, y si éstas no bastassen hecharse un rediezmo de todos los frutos que se cogieren» en Montblanc. Como veremos, estas mejoras sanitarias llevarían a la progresiva disminución de la prevalencia de estas enfermedades. En segundo lugar, analizada la calidad de las enfermedades y dada la proximidad del verano, se aconsejaba aplicar remedios eficaces dirigidos a la curación de los enfermos y a «preservar la salud de los que oy se mantienen sanos». Así, para «assistir con segura providencia a los dolientes pobres de solemnidad», los médicos aseguraban que se debían establecer tres hospitales: uno en Montblanc, donde también se curarían los enfermos pobres de Blancafort, la Guàrdia dels Prats y Lilla; otro en Sarral, que acogería a los de Solivella, Pira, Ollers y Barberà; y otro en la Espluga de Francolí, sólo para sus vecinos. Los hospitales se tenían que formar «a costa de los mismos Pueblos y con el menor dispendio possible de la Real Hazienda». Pero dada la dificultad de contribuir a causa de las enfermedades, solicitaron la suspensión de «la exacción del catastro y denás contribuciones», así como una orden para que no «se les execute en común, ni en particular por ninguno de sus acrehedores» hasta el restablecimiento de la salud de la población, con el fin de poder subvencionar los hospitales. A esto se debería de añadir el rediezmo de todos los frutos que se cogían en todos los pueblos, a excepción de Montblanc, así como el producto de la caridad de los prelados y de los señores jurisdiccionales de los pueblos ${ }^{24}$.

El informe de la comisión fue entregado al Consejo de Castilla, que se conformó con todas las recomendaciones ofrecidas, según indica la Real Orden de 3 de julio de 1727, en la que se dictaba un período de dos años de aplazamiento del cobro del catastro y de otras cargas con la justificación de la curación de las enfermedades. Además, se ordenaba escribir a las justicias y eclesiásticos de la Espluga, Montblanc y Sarral «poniéndoles en la inteligencia de lo que deven a la Real Benignidad de S.M. en la suspensión del Catastro y demás beneficios que les dispensa su Paternal clemencia». Se cumplía así el deber de los gobernantes de proteger a los pobres en tiempo de crisis. De esta forma, no sólo se legitimaba el gobierno del Rey y de sus representantes, sino también una tradición, una moral o deber del Rey hacia sus súbditos de afirmación y muestra de buen padre protector ${ }^{25}$.

24. ACA, RA, Consultas, Reg. 139, B. 12/4/1727, ff. 77r-85r, Informe de la RA al Rey.

25. ACA, RA, Consultas, Reg. 139, B. 21/8/1727, ff. 167r-168v, Respuesta de la RA a las cartas del Consejo de Castilla; y Villetes, Reg. 370, Madrid, 3/8/1727, ff. 72v-74v, Consejo de Castilla a Capitán General. 
Veamos a continuación el espacio político en el que se desarrollaron las medidas arbitradas. Esto es, analizar los intereses económicos y políticos que afloran en una situación crítica. En primer lugar, la actuación de las autoridades reales se dirigió a persuadir a los señores jurisdiccionales y prelados eclesiásticos de su obligación a contribuir a la ayuda de los enfermos y a practicar la caridad, «aplicando por lo menos para ello los diezmos y rentas que gozavan en los mismos pueblos». Las autoridades invocaban aquí una obligación moral de caridad y piedad cristiana hacia los pobres, pero a la vez aseguraban a los señores que no habría cambios en el orden establecido sino una interrupción temporal ya que, una vez superadas las enfermedades, los municipios tendrían que pagar los atrasos. La aplicación de tales propósitos no fue fácil. En efecto, el Arzobispo de Tarragona se excusó ante el primer llamamiento real alegando que ya estaba asistiendo a los enfermos de las zonas arroceras del Campo de Tarragona. De hecho, tan sólo el Abad de Santes Creus accedió a que el producto del diezmo de la Guàrdia se aplicase a uno de los hospitales proyectados. Desde el punto de vista de las responsabilidades, la actitud negativa de las autoridades jurisdiccionales a dejar de percibir las rentas nos llevaría a cuestionar, en este caso, la validez de las tradiciones de caridad locales ${ }^{26}$. En este sentido, las denuncias de los comunes contra los intentos de los señores de cobrar sus derechos aparecen a pesar de la real orden de suspensión: en el mes de septiembre de 1727, determinados eclesiásticos de Montblanc «se han puesto a cabrevar de suerte que importarán todas las cabrevaciones más de $4.000 \mathrm{li}$ bras». Más adelante, las curias eclesiásticas de Tarragona y del decanato de Montblanc intentaron introducir «muchos gastos a particulares de dicha Villa sobre censales que haze aquel Común». Estas curias continuaron litigando e, incluso, ordenaron un «decreto de execución conminándoles con excomunión». Los municipios reclamaron su derecho contra estos intentos que pasaban por alto la vigencia de la orden de suspensión de los pagos. La Real Audiencia actuó siempre ante estas quejas empleando una táctica persuasiva que se basó en recordar que «no es justo que temerariamente quieren atropellar a los que S.M. indulta (...), imposibilitándoles su recobro, con que después por junto poder satisfacer al Rey y demás acrehedores» ${ }^{27}$.

Una siluación semejante se halla en el caso de la percepción del catastro y la actitud tomada por la Intendencia de Cataluña. Tan sólo un año después de la suspensión real, la villa de Sarral se encontraba forzada por la caballería, por el ejército borbónico, con dos cartas de pago de 30.522 reales y 10.174 reales enviadas por el Intendente. También Montblanc se halló en 1728 con el libramiento de la primera tercia del catastro del año anterior y en el año 1729 con cartas de pago de las tercias correspondientes a 1728 y 1729. A estas quejas, los pueblos añadían que, si bien el Intendente les forzaba a pagar a partir del 31 de julio de 1729 los atrasos y el catastro pre-

26. Sólo tres de Ios diez pueblos eran de jurisdicción real: Montblanc, Blancafort y Lilla. Pira, Ollers y Barberà eran del Prior de Cataluña de la Religión de San Juan; la Espluga era de don Antoni Puigdorfila. Comendador de San Juan; Sarral del Conde de Vallcabra; Solivella de don Joan de Llorach y Guàrdia dels Prats del monasterio de Santes Creus. El Prelado eclesiástico de todos los pueblos era el Arzobispo de Tarragona. ACA, RA, Consultas, Reg. 139, B. 21/8/1727, ff. 167r-168v, Informe de la RA; y Villetes, Reg. 380, B. 15/11/1727, ff. $111 \mathrm{v}-112 \mathrm{v}$. Informe del Capitán General sobre las ayudas de los señores y prelados.

27. ACA. RA. Consultas, Reg. 139, B. 4/9/1727, ff. 186r-187r; Reg. 141, B. 19/7/1728, ff. 26v-27r; Reg. 142, B. 16/5/1729, f. 210rv; Villetes, Reg. 370, B. 28/8/1727, ff. $78 \mathrm{rv}$. 
sente, el Rey les había prometido hacer una nueva medición de todas las tierras de los diez pueblos por considerar excesivas las 15.708 libras que anualmente debían pagar. La Real Audiencia fue enérgica durante el tiempo de la suspensión al mandar la retirada del auxilio militar para forzar el cobro, al recriminar la actitud del Intendente contra la orden real y al pedir la nueva medición de las tierras. A pesar de esto, la Audiencia derivó las súplicas de las poblaciones al Rey cuando se estaba cerca de la finalización del plazo de suspensión, eludiendo así su responsabilidad de gobierno en el Principado ${ }^{28}$.

La percepción del rediezmo de los frutos de todos los pueblos y la conducción del agua de la Fuente de la Pasquala a Montblanc también se añadieron a la anterior tónica conflictiva. En cuanto al rediezmo de Montblanc, su arrendamiento no comenzó a ser productivo hasta el primero de mayo de 1728. A pesar de llegar el año anterior a un consenso sobre su control dual -en manos del plebano de Montblanc y de un «patricio» de la villa, Salvador Batlle-, los fraudes en los pagos del primer año lo dejaron sin arrendatario. Y ello a pesar de la actitud de la Audiencia que advertía que «a los que no quisieren consentir en el rediezmo se les impondrá una talla correspondiente a él, para que contribuyan igualmente todos a la importante fábrica de la referida fuente». La Audiencia apelaba a la necesidad de la obra y a su utilidad general a la hora de obligar a pagar a todos aquellos eclesiásticos y terratenientes que tuvieran propiedades en la villa, así como a los menestrales «a proporción de los efectos gananciales que tengan». De hecho, se trataba de una solidaridad forzada, ya que si bien el Arzobispo de Tarragona accedió finalmente al pago, no lo hizo así la ciudad de Tarragona, pues no hallaba beneficio en el acto. También tuvo problemas el arrendatario del rediezmo de los frutos de los otros nueve pueblos, Raimon Ortells, quien se quejó de «no querer los procuradores de el Comendador de la Espluga y lugar de Barberà del orden de San Juan, el cura de dicha villa y el de Blancafort, y demás eclesiásticos pagar el rediezmo de sus frutos por decir gozar de la inmunidad eclesiástica». La Audiencia respondió que esto no era contra tal inmunidad, «antes bien muy conforme a ella y a la christiana piedad de los eclesiásticos», consiguiendo finalmente el consentimiento del Gran Prior de Cataluña ${ }^{20}$.

En Montblanc, el inicio de las obras de conducción de agua se retrasó hasta el mes de septiembre de 1727 y éstas no acabarían hasta mayo de 1729, rematándose en septiembre de dicho año. Entre los motivos de los atrasos hallamos de nuevo la oposición de la ciudad y capítulo de Tarragona, quienes aseguraban que el agua de la fuente les pertenecía y por ello intentaron forzar un pleito y ordenaron mandatos penales contra Montbianc por apropiación del agua con el fin de paralizar la conducción. La actitud de la Audiencia fue aquí la de «pacificar la dependencia». En este sentido, la Audiencia escribió cartas a la ciudad, al síndico del capítulo y al Arzobispo de Tarragona para conseguir su cesión. En aquéllas se les manifestaba «quanto será del servicio de Dios y del Rey, y Beneficio Común (...) el que se execute la obra» para la curación de

28. ACA, RA, Consultas, Reg. 141, B. 14/7/1728, ff. 20v-21v; B. 24/7/1728, if. 46v-49r; Reg. 142, B. 6/5/1729, ff. 183r-184r; Reg. 143, B. 11/6/1729, ff. 3v-5v.

29. ACA, RA, Consultas, Reg. 139, B. 16/5/1727, ff. 116r-117r; B.19/6/1727, ff. 251v-253r; B 16/7/1727, ff. 149r-150v; B. 23/8/1727, ff. 155v-157r; Reg. 141, B. 22/7/1728, ff .45v-46v y B. 12/9/1728, f. 92r. Villetes, Reg. 371, B. 31/8/1728, ff. 1 86rv. Informes de la Real Audiencia. 
los enfermos. Pidiéndoles que no litigasen en materia en la que «se interesa principalmente la caridad christiana y el Bien Común del Principado por la Pública Salud de los vecinos de aquellos parages». Pero también les amenazaba diciendo que las resoluciones contrarias del capítulo serían «del desagrado de S.M. y desaprobación de Su Excelencia». Finalmente, se consiguió un trazado conciliador ${ }^{30}$.

Parece probable que la declinación de las enfermedades se produjese gracias a terraplenar las balsas y a la conducción de agua, pero también fue importante el dificultoso establecimiento de hospitales para asistir a los pobres enfermos. La escasez de las rentas de los pueblos afectados hizo que los regidores de Montblanc, Sarral y la Espluga se obligasen con sus propios bienes y los de sus Universidades a mantener y curar a setenta y siete enfermos pobres en los tres hospitales. Sin embargo la Audiencia, a pesar de la oposición del ministro comisionado y de los médicos de las villas, ante la falta de liquidez, la multitud de enfermos y la insuficiencia de los hospitales, comprendió que «no se podía lograr el fin de medicarse aquella epidemia». Por tanto, ordenó que no entrasen más enfermos y que a los enfermos pobres y sin recursos para restablecerse «se les assistiesse en sus casas para remediar su miseria por el Bayle y Regidores de Montblanch, Espluga y Sarral». Así quedó el asunto desde el mes de enero de 1728. El Rey se interesó entonces por el éxito de las medidas y la Audiencia aseguró que no sólo se había parado el gasto de los hospitales, pues era más barata la asistencia en las casas y así con el remanente se podrían pagar el catastro y las deudas de los pueblos, sino que, además, de esta forma las enfermedades casi habían desaparecido, ya que sólo quedaban 218 enfermos en toda la Conca, de los que únicamente 38 eran pobres. No podemos discutir aquí si la medida de la Audiencia fue más acertada que el voto de Roxas y de los médicos a favor del mantenimiento de los hospitales. En cualquier caso, parece claro que las enfermedades aminoraron a medida que las órdenes dictadas avanzaron, a pesar de las dificultades, de forma conjunta en el tiempo y, también, que el suministro de alimentos durante la convalecencia fue fundamental para el restablecimiento de los enfermos. Por lo tanto, el enfrentamiento entre los ministros de la Real Audiencia manifiesta que las medidas de lucha dictadas por la Corona tuvieron un éxito relativo a causa de los obstáculos constantes que se presentaron y de la consecuente prolongación en la recuperación de los enfermos.

En definitiva, esto era producto de la incapacidad de aquel Estado para hacer frente a los múltiples problemas que surgieron, básicamente de carácter jurisdiccional y económico. En este sentido, cabe recordar que el gasto general sería pagado por los propios afectados por las enfermedades, durante la aplicación de las medidas y una vez restablecidos y pasado el plazo de excepción decretado por la Corona. Este fue, en conclusión, el juego de deberes y obligaciones morales con los que el Estado del siglo XVIII tomó la parte de responsabilidad política que le correspondía ${ }^{31}$.

30. ACA, RA, Consultas, Reg. 139, B. 17/5/1727, ff. 118rv; B. 12-14-23/8/1727, ff. 155v-157r, 16Iv164r, 169r-170r; B. 12/9/1727, ff. 188v-190r; Reg. 142, B.20/5/1729, ff. 221v-222r; Reg. 143, B. 21 $28 / 7 / 1729$, ff. $75 \mathrm{rv}, 92 \mathrm{v}-93 \mathrm{r}$ y $95 \mathrm{rv}$. Informes de la Real Audiencia.

31. Son muy interesantes las razones aducidas por los ministros de la Audiencia en defensa de las diferentes opciones, asi como las instrucciones sobre establecimiento y formación de los hospitales, véase: ACA, RA, Consultas, Reg. 139, B. 24/10/1727, ff. 25rv; Reg. 140, B. 18/4/1728, If. I43r-144r; Reg, 142, B. 23/4/1729, ff. 140r-152r i 152r-161r, i B. 6/6/1729, ff. 257v-260r. 


\section{BIBLIOGRAFÍA}

ANDREU, J., 1986, Economia i Societat a Reus durant la crisi de l'Antic Règim, Associació d'Estudis Reusencs, Reus.

ANDREU, J., 1994, El Camp de Tarragona i el Priorat durant els segles XVIII i XIX. Les Bases demogràfiques, agràries i comercials de l'expansió setcentista, Tesis de doctorado inédita, UAB, Bellaterra.

ARRANZ, M., 1985, «Epidèmies i crisis agràries a la Catalunya Nova en el decenni de 1720: l'actitud de les autoritats borbòniques», Aplec, 5, 193-216.

CALBET, J.M. y VALLRIBERA, P., 1990, Medicina $i$ Societat a l'Espluga de Francolí (segles XVIII $i$ XIX), UB, Barcelona.

CARMICHAEL, A.G., 1985, «Infection, Hidden Hunger, and History», en ROTBERG, R.I. y RABB, T.K. (eds), Hunger and History. The Impact of Changing Food Production and Consumption Patterns on Society, CUP, Cambridge, 51-66.

CASANOVAS, S., 1978, Memòries d'un pagès del segle XVIII, Curial, Barcelona.

FELIU, G., 1991, Precios y salarios en la Cataluña Moderna, Banco de España, Madrid.

FERRER, LI., 1989, «Notas sobre las estructuras agrarias catalanas en el siglo XVIII», en Estructuras Agrarias y Reformismo Ilustrado en la España del siglo XVIII, Ministerio de Agricultura, Madrid.

GARRABOU, R., 1970, «Sobre la formació del mercat català en el segle XVIII», Recerques, $1,83-121$.

GRAU, J.M.T., et al., 1987, «La població de la Conca de Barberá entre la crisi de mitjan XVII $i$ les epidèmies del 1725-1727», Aplec, 8, 169-180.

GRAU, J.M.T., 1990, Població i lluita contra la mort a Montblanc (s. XVIII), Diputació de Tarragona, Tarragona.

GUAL, V., 1994, «Les crisis demogràfiques de la primera meitat del segle XVIII a la Conca de Barberà», Aplec, 12, 105-123.

IGLÉSIES, J., 1974, Estadístiques de Població de Catalunya. El primer vicenni del segle XVIII, 3 vols., Fund. S.Vives Casajuana, Barcelona.

JORDI, R., 1981, «Notas sobre terapéutica hospitalaria usada en una epidemia de Montblanc. Año 1725», Circular Farmacèutica Butlletí Informatiu, XII, 138, 69-73.

JULIANO, D., 1987, «Evolució demogràfica a Barberà de la Conca», Aplec, 7, 47-1 15.

LIVI-BACCI, M., 1988, Ensayo sobre la historia de la demografía europea. Población y alimentación en Europa, Ariel, Barcelona.

LLIMARGUES, J., 1989, «Els sistemes locals de previsió de l'escassetat a les acaballes de l'Antic Règim (Catalunya i Provença, 1750-89)», en El Jacobinisme. Reacció i Revolució a Catalunya i Espanya, 1789-1837, UAB, Bellaterra, 111-122.

MATEU, E., 1987, Arroz y Paludismo, Alfons el Magnànim-IVEI, Valencia.

NADAL, J., 1988, La población española (siglos XVI a XX), Ariel, Barcelona.

NAVARRO, L.J., 1984, «Aproximación a un estudio de la agricultura del Corregimiento de Tarragona en la primera mitad del siglo XVIII, a través del Real Catastro», en Congreso de Historia Rural, siglos XV al XIX, UCM-Casa de Velázquez, Madrid.

PÉREZ MOREDA, V., 1980, Las crisis de mortalidad en la España interior. Siglos XVI-XIX, Siglo XXI, Madrid. 
PÉREZ MOREDA, V., 1988, «Hambre, mortalidad y crecimiento demográfico en las poblaciones de la Europa Preindustrial», Revista de Historia Económica, 3, 709-735.

PESET, M. y PESET, J.L., 1972, «Cultivos de arroz y paludismo en la Valencia del siglo XVIII», Hispania, XXXII, 275-375.

PORTA, J.M., 1979, «Una aproximació a la població de Montblanc: estat de la qüestió (s.XIV, XV, XVI i XVIII)», en ler Col-loqui d'Història del Camp de Tarragona, Conca de Barberà i Priorat, Tarragona, 41-47.

PORTA, J.M., 1986, La vila de Montblanc en el segon quart del segle XVIII, Generaltitat de Catalunya, Barcelona.

SALES, N., 1980, Història dels Països Catalans-III, Edhasa, Barcelona.

SALES, N., 1989, Els segles de la decadència (segles XVI-XVIII), Història de Catalunya-Edicions 62, Barcelona.

SOLER, S., 1994, Memòries d'una família pagesa: els Anglada de Fonteta (segles XVIIXVIII), Ajuntament de La Bisbal, La Bisbal.

TELLO, E, 1995, Cervera i la Segarra al segle XVIII. En els origens d'una Catalunya pobre, Pagès editors, Lleida.

THOMPSON, E.P., 1994, Costumbres en Común, Crítica, Barcelona.

VICEDO, E., 1991, Les terres de Lleida i el desenvolupament català del Set-cents. Producció, propietat $i$ renda, Crítica, Barcelona.

VILAR, P., 1962, La Catalogne dans l'Espagne Moderne, 3 vols., Sevpen, París.

ZARZOSO, A., 1994, Prevenció epidèmica i salut pública a la Barcelona del segle XVIII, Trabajo de investigación de doctorado, UPF-IUHJVV, Barcelona. 


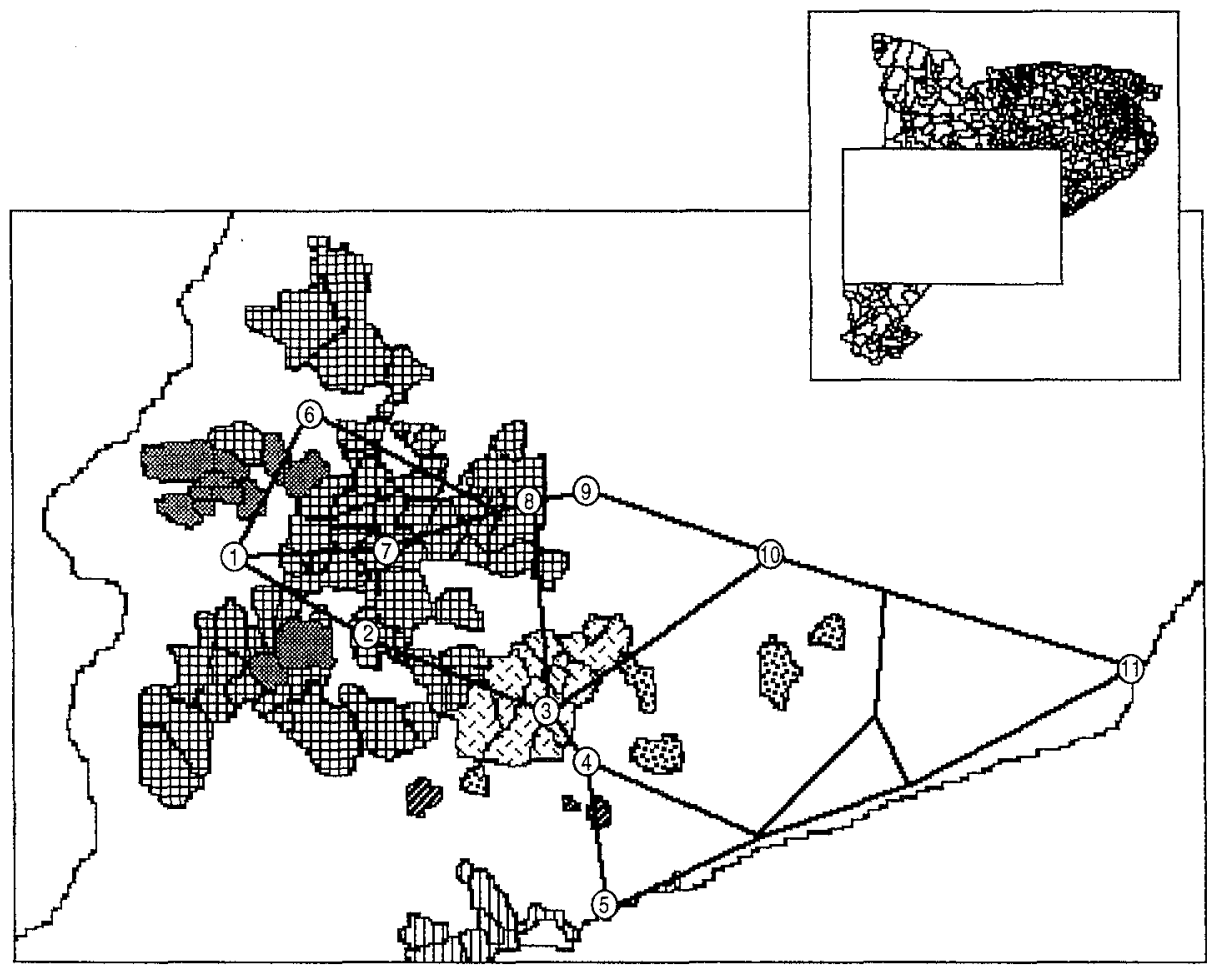

W1719-1723, enfermedades

Verano-otoño 1722, enfermedades

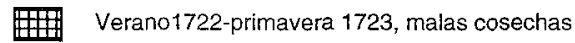

Septiembre 1724-verano1725, enfermedades

$\therefore$ Verano1724-1729, enfermedades

III] Verano1727-principios 1729, enfermedades
1.- Lleida

2.- Borges Blanques, Les

3.- Montblanc

4.- Valls

5.- Tarragona

6.- Balaguer

7.- Mollerussa

8.- Tàrrega

9.- Cervera

10.- Igualada

11.- Barcelona 